\title{
A High Performance Flexible Pressure Sensor Realized by Overhanging Cobweb-like Structure on a Micropost Array
}

Weiguan Zhang,,${ }^{1,2}$ Yan Xiao, ${ }^{2}$ Yu Duan, ${ }^{2}$ Ning Li, ${ }^{2}$ Linlin $\mathrm{Wu},{ }^{2}$ Yan Lou,,${ }^{1}$ Hao Wang,,${ }^{*}$ and Zhengchun Peng ${ }^{1,2 *}$

1 Guangdong Provincial Key Laboratory of Micro/Nano Optomechatronic Engineering, College of Mechatronics and Control Engineering, Shenzhen University

Shenzhen 518060, China

${ }^{2}$ Key Laboratory of Optoelectronic Devices and Systems of Ministry of Education and Guangdong Province, College of Physics and Optoelectronic Engineering Shenzhen University, Shenzhen 518060, China

\section{Corresponding Author}

E-mail:

Zhengchun Peng: zcpeng@ @zu.edu.cn;

Hao Wang:whao@szu.edu.cn; 


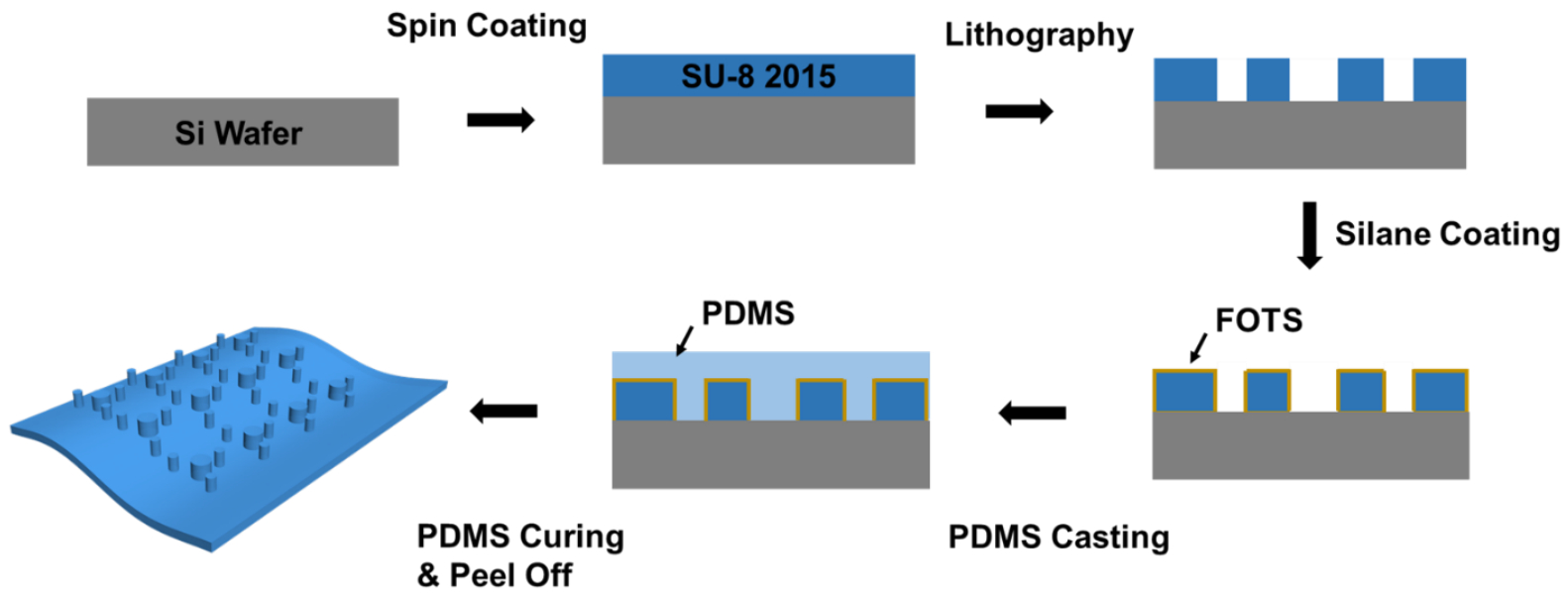

Figure S1. The detailed fabrication process of the PDMS substrate with micropost array.

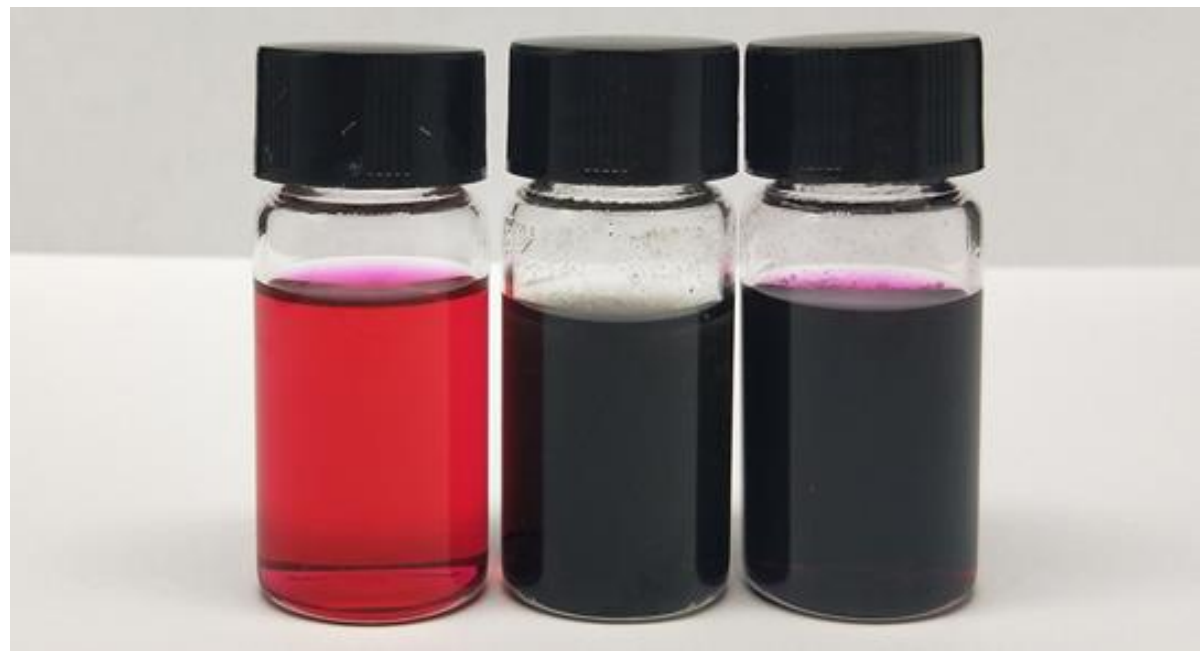

Figure S2. Optical photograph of ZnOEP, CNT, and ZnOEP/CNT hybridized solution. 

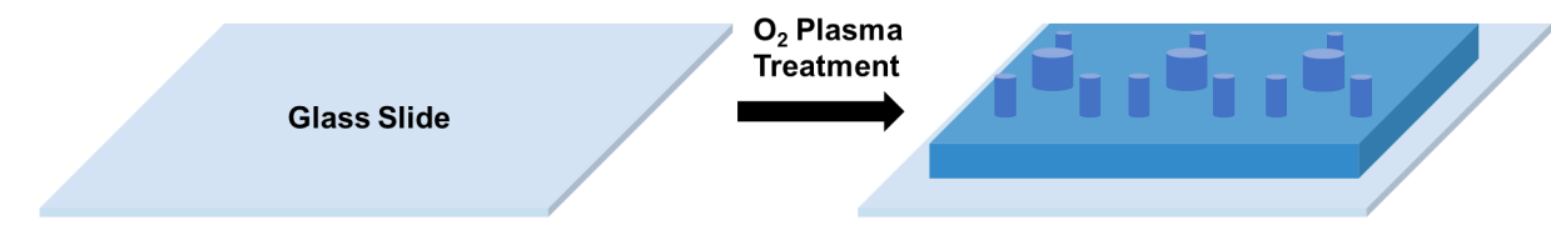

Dip-coating of

ZnOEP/CNT Solution

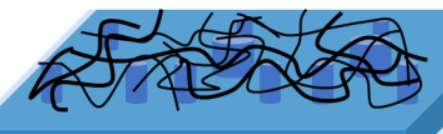

Self-assembly
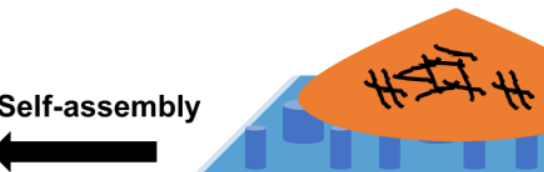

Figure S3. Fabrication process of flexible pressure sensor with cobweb-like hybrid on micropost

array.
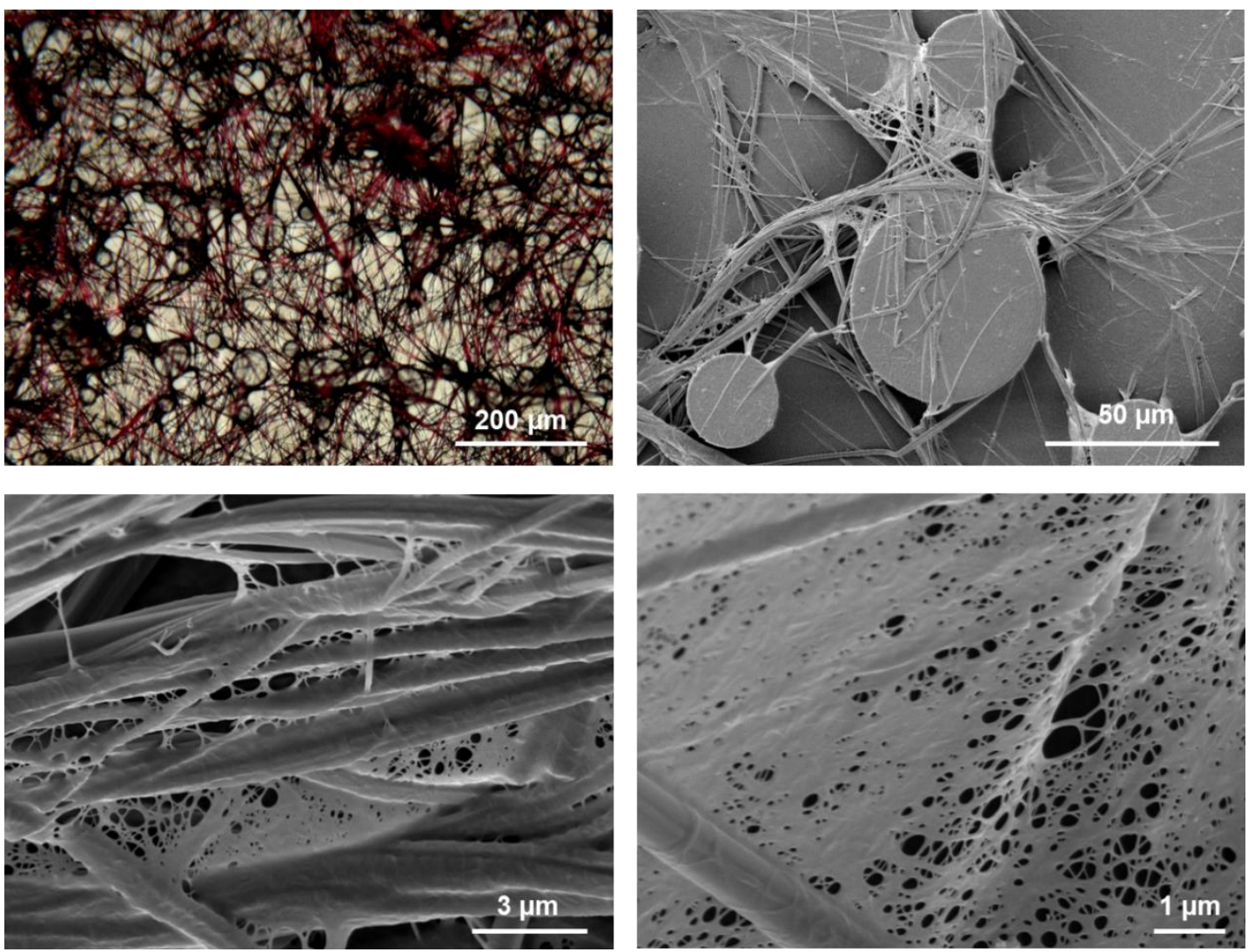

Figure S4. Optical and SEM images of the cobweb-like hybrid material. 

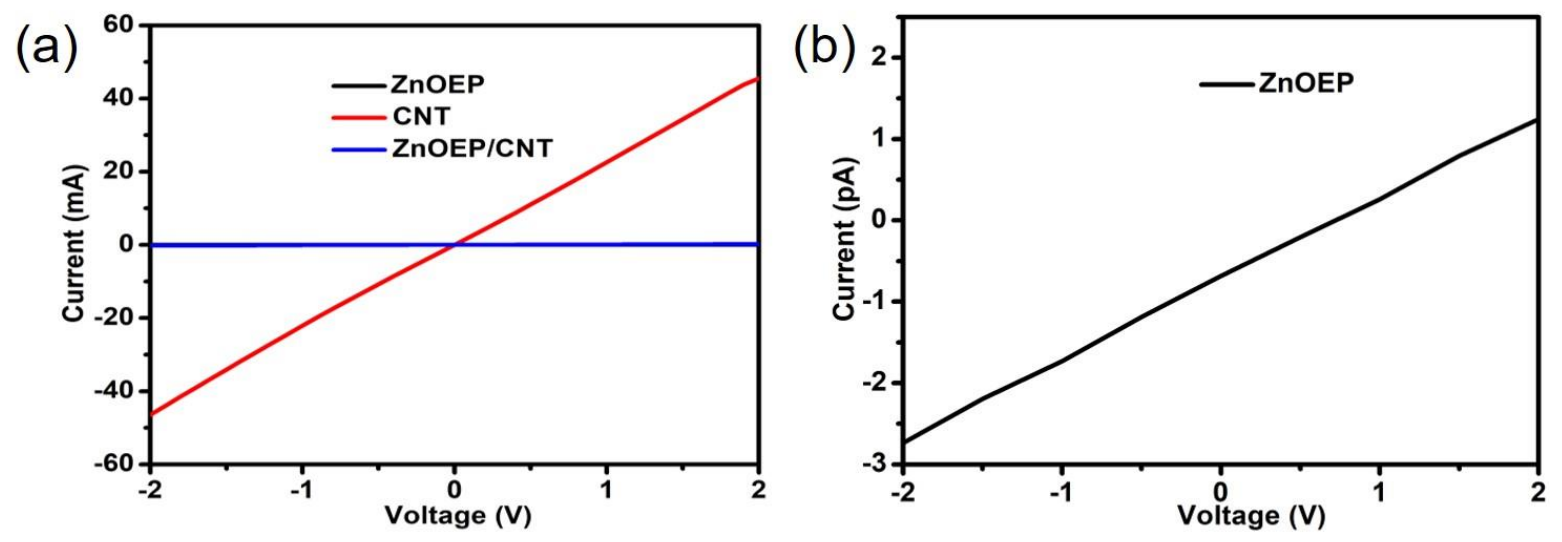

Figure S5. (a) the I-V curves of pure ZnoEP, CNT and ZnOEP/CNT hybrid, (b) the magnification of pure $\mathrm{ZnOEP} \mathrm{I-V} \mathrm{curve.}$ 